\title{
BIBLIOGRAPHY
}

1. V. F. Cowling, W. Leighton, and W. J. Thron, Twin convergence regions for continued fractions, Bull. Amer. Math. Soc. vol. 50 (1944) pp. 351-357.

2. J. Dieudonne, La theorie analytique des polynomes d'une variable, Memorial des Sciences Mathématiques, vol. 93, Paris, 1938.

3. W. Leighton and W. J. Thron, Continued fractions with complex elements, Duke Math. J. vol. 9 (1942) pp. 763-772.

The University of Kentucky

\section{NORMS OF MATRIX TYPE FOR THE SPACES OF CONVERGENT AND BOUNDED SEQUENCES}

\section{ALBERT WILANSKY}

Let, as usual, $x=\left\{x_{n}\right\} \in(c)$ mean that $x$ is a convergent sequence. We write $\|x\|=\|x\|_{A}=\sup _{n}\left|\sum_{k=1}^{n} a_{n k} x_{k}\right|=\sup _{n}\left|A_{n}(x)\right|$, where $A$ $=\left(a_{n k}\right)$ is a matrix of complex numbers. By the ordinary norm of $x$ we shall understand $|x|=\sup _{n}\left|x_{n}\right|$.

Problem 1. What conditions on $A$ are necessary and sufficient that (c) be a Banach space with this norm?

The first result is that $\|x\|<\infty$ for all $x \in(c)$ if and only if $\|A\|$ $=\sup _{n} \sum_{k=1}^{\infty}\left|a_{n k}\right|<\infty$ (essentially due to Toeplitz, 1911), and this is true if and only if $\|x\|<\infty$ for all $x \in(m)$, the space of bounded sequences.

We shall assume that $A$ is normal, that is, that $a_{n k}=0$ for $k>n$, while $a_{n n} \neq 0$. (A remark on reversibility will be appended.) Finally, let $(A)$ be the class of sequences $x$ such that $A x=\left\{A_{n}(x)\right\}$ is convergent.

Theorem 1. Let $A$ be normal, $\|A\|<\infty$, then for $(c)$ to be a Banach space it is sufficient that $\left\|A^{-1}\right\|<\infty$. The metric will then be equivalent to the ordinary metric. The condition is not necessary (even if $A$ is conservative).

(A conservative matrix-one with $(A) \supset(c)$-must satisfy $\|A\|$ $<\infty$, but not conversely.)

Assume that $A$ satisfies the hypotheses. For $x \in(A), y=A x$, we deduce that $\left|x_{n}\right| \leqq\left\|A^{-1}\right\| \cdot \sup _{n}\left|y_{n}\right|=\left\|A^{-1}\right\| \cdot\|x\|$, and conclude: (1) $(A) \subset(m) ;(2) L_{n}(x)=x_{n}$ defines an additive continuous functional $L_{n}$ on $(A)$.

Presented to the Society, February 24, 1951 under the title On norms of matrix type for $(c)$ and $(m)$; received by the editors October 16, 1950. 
We now show that $(A)$ is complete using the ordinary norm. A Cauchy sequence of elements of $(A)$ must have a limit in $(m)$ by (1); moreover since (3) $\|x\|_{A} \leqq\|A\| \cdot|x|$, it is a Cauchy sequence using the norm $\|\cdot\|_{A}$, and thus has a limit in $(A)$ using this norm. (That $A$ is complete using $\|\cdot\|_{A}$ is well known. It follows from the equivalence of $(A)$ with $(c)$, using the ordinary norm in (c).) By (2) these two limits are the same so that $(A)$ is complete as stated.

Next, for $x \in(A)$ let $U(x)=A x \in(c)$. This is an additive 1-1 operation from $(A)$ onto $(c)$. Using the ordinary norm in $(A),\|\cdot\|_{A}$ in $(c)$, it is clear that $U$ is continuous since $\|U(x)\|=\sup _{n}\left|A_{n}(A x)\right| \leqq\|A\|^{2}$ $\cdot|x|$. Similarly $\left|U^{-1}(y)\right| \leqq\left.\left\|A^{-1}\right\|\right|^{2} \cdot\|y\|$, so that $U^{-1}$ is continuous. (Its continuity is also a consequence of the general theorem on inverses of maps of Banach spaces, see Banach, Théorie des opérations linéaires, Theorem 5, p. 41.) Thus $(A)$ and $(c)$ with their assigned metrics are homeomorphic, and, $(A)$ being complete, so is $(c)$. This completes the proof of the direct statement. The equivalence of the metrics follows from Banach, loc. cit., Theorem 6, p. 41, taking account of (3) above. Before completing the proof we state, for the sake of completeness, the following theorem.

TheOREM 2. If, in Theorem 1, $A$ is assumed conservative, then actually $(c)=(A)$ is equivalent to the sufficient condition.

This is the main result of the author's paper appearing in Bull. Amer. Math. Soc. vol. 55 (1949) p. 914. We shall refer to this paper as [B].

To prove the last statement in Theorem 1 we define $A$ by $a_{n n}=1$, $a_{n, n-1}=2, a_{n k}=0$ otherwise. Then $\left\|A^{-1}\right\|=\infty$, either by direct computation, or by $[\mathrm{B}]$ since $(A)$ contains the sequence $\left\{(-2)^{n}\right\}$. We shall designate this sequence as $u$. We now show $(c)$ to be complete. For an arbitrary sequence $x$ the identity $x_{n}=(-2)^{n} \sum_{k=1}^{n}(-1 / 2)^{k} A_{k}(x)$ can be easily checked. Now for $x \in(A)$, the series in this identity converges, say to $f(x)$. Then $x_{n}-(-2)^{n} f(x)=-(-2)^{n} \sum_{k=n+1}^{\infty}(-1 / 2)^{k} A_{k}(x)$ $=-\sum_{k=1}^{\infty}(-1 / 2)^{k} A_{k+n}(x)$, and this latter series is uniformly convergent, thus, as a function of $n$, is a convergent sequence. Call it $c$. Thus every $x \in(A)$ satisfies $x=c+u f(x)$ where $c$ is convergent and so $(A)$ is the smallest linear space including $(c)$ and the sequence $u$. (This result shows an application of the author's "orthogonal functionals," see Trans. Amer. Math. Soc. vol. 67 (1949) p. 64.) Now $f(x)$ $=\lim x_{n} /(-2)^{n}=0$ for $x \in(c)$, while $f(u)=1$, also $f$ is continuous. Thus $(c)$, the set of zeros of $f$, is closed.

The defect in Theorem 1 due to the falsity of the converse can be remedied. In order that $\left\|A^{-1}\right\|<\infty$ for a conservative matrix $A$ it is 
necessary [B] that the columns of $A-1$ be convergent sequences, and this is just the missing condition for the converse of Theorem 1. Let $\bar{a}_{n k}$ be a typical entry in $A^{-1}$.

Theorem 3. Let $A$ be normal, $\|A\|<\infty$, and suppose that $\lim _{n} \bar{a}_{n k}$ exists for each $k$. Then for $(c)$ to be a Banach space it is necessary that $\left\|A^{-1}\right\|<\infty$.

As above, we define $L_{n}(x)=x_{n}$, but now we are able to determine $\left\|L_{n}\right\|$. Since $L_{n}(x)=\sum_{k} \bar{a}_{n k} A_{k}(x)$, we obtain $\left\|L_{n}\right\| \leqq \sum_{k}\left|\bar{a}_{n k}\right|$; next, with fixed $n$, set $y_{k}=\operatorname{sgn} \bar{a}_{n k}$, then with $x=A^{-1} y$ we have $\|x\|=1$, $x \in(c)$, and $L_{n}(x)=x_{n}=\sum_{k=1}^{n}\left|\bar{a}_{n k}\right|$, and so $\left\|L_{n}\right\|=\sum_{k}\left|\bar{a}_{n k}\right|$. Since $\sup _{n}\left|L_{n}(x)\right|<\infty$ for each $x$, it follows from the Banach-Steinhaus theorem (Banach, loc. cit., Theorem 5, p. 80) that $\left\|L_{n}\right\|<M$ for some $M$ and all $n$. The result follows from the definition of $\left\|A^{-1}\right\|$ and the evaluation of $\left\|L_{n}\right\|$.

TheOREM 4. Let $A$ be normal, conservative, such that (c) is a Banach space. Then that $\left\|A^{-1}\right\|<\infty$ it is necessary and sufficient that $\lim _{n} \bar{a}_{n k}$ exist for each $k$.

Sufficiency was proved in Theorem 3 while necessity is part of the result of $[\mathrm{B}]$.

Problem 2. This is the same as Problem 1 with $(m)$ instead of $(c)$.

We find a close parallel in the theory, and one-half of a connecting result (Theorem 5).

TheOREM 1'. We may replace $(c)$ by $(m)$ in Theorem 1.

Let $K$ be the set of all sequences $x$ such that $\|x\|<\infty$, that is, $A x$ is a bounded sequence. As in the proof of Theorem 1 we deduce that $K \subset(m)$, hence $K=(m)$. To $x \in K$ make correspond $A x \in(m)$. This is an equivalence between $K$ and $(m)$, where the ordinary norm is used in $(m)$. Thus $K$ is complete and so is $(m)$ since $(m)=K$.

The same counterexample used for Theorem 1 works here. Trivial adjustments in the argument show that $K$ is the smallest linear space including $(m)$ and the sequence $u$, and that $(m)$ is closed.

For the analogue of Theorem 3 we need a preliminary result.

Let us, for the sake of brevity, proceed with slightly less generality, namely with the assumption that $A$ is actually conservative. We shall append a remark on a method of restoring the generality.

Theorem 5. If $(m)$ is a Banach space, so is (c).

Our assumption is that $A$ is normal, conservative. Let $X$ be the 
intersection of $(A)$ and $(m)$. It is closed under the given conditions. The reasoning of $[B]$ from the last paragraph of p. 915 to the end of the paper shows that $X=(c)$. Thus $(c)$ is also complete.

Theorem 3 '. Let $A$ be normal, conservative, and suppose that $\sup _{n}\left|\bar{a}_{n k}\right|<\infty$ for each $k$. Then for $(m)$ to be a Banach space it is necessary that $\left\|A^{-1}\right\|<\infty$, thus that $(A)=(c)$.

Notice the expected change from convergent columns to bounded columns. Without this change the result is trivial from Theorems 5 and 3. The proof is identical with that of Theorem 3 save that the sentence " $x \in(c)$ " is replaced by " $x \in(m)$."

APPENDIX 1. In connection with Theorem 3 we can state the result: If $\|A\|<\infty$ and $A$ is not conservative, then $A$ must sum divergent sequences. For if $(A) \subset(c), A^{-1}$ is conservative and the result of [B] applies. Of course we cannot conclude that $A$ must sum unbounded sequences. Indeed, it cannot if $\left\|A^{-1}\right\|<\infty$.

2. If $A$ is co-regular and $(c)$ is a Banach space, then $X$, defined in the proof of Theorem 5 , is equal to $(c)$. For definitions and results see the Transactions paper quoted above. Under these conditions, $(c)=\overline{(c)}=\bar{X} \supset X \supset(c)$ and so $X=(c)$.

3 . It is an open question whether $(c)$ may be complete while $(m)$ is not.

4. To obtain Theorems 5 and $3^{\prime}$, assuming $\|A\|<\infty$, we need a generalization of the result of Agnew used at the end of [B] to read: If $\|A\|<\infty$, then either $\left\|A^{-1}\right\|<\infty$ or $X=(A) \cap(m)$ is not separable using the ordinary norm.

5. The arguments of this paper will apply if $A$ is assumed only reversible, rather than normal. The methods are made clear in [B] and, without adding any essential difficulties to the proofs, such generality only complicates the notation.

LEHIGH UNIVERSITY 